Erratum

\title{
Erratum: Liu, Y.-Z.; Jiang, X.-K.; Cao, W.-F.; Sun, J.-Y.; Gao, F. Detection of Thrombin Based on Fluorescence Energy Transfer between Semiconducting Polymer Dots and BHQ-Labelled Aptamers. Sensors 2018,
}

\section{8,589}

\author{
Yizhang Liu 1,2,*, Xuekai Jiang ${ }^{2}$, Wenfeng Cao ${ }^{2}$, Junyong Sun ${ }^{2}$ and Feng Gao ${ }^{2, *}$ \\ 1 Department of Food and Environmental Engineering, Chuzhou Vocational and Technical College, \\ Chuzhou 239001, China \\ 2 Laboratory of Functionalized Molecular Solids, Ministry of Education, \\ Anhui Key Laboratory of Chemo/Biosensing, Laboratory of Optical Probes and Bioelectrocatalysis (LOPAB), \\ College of Chemistry and Materials Science, Anhui Normal University, Wuhu 241000, China; \\ jiangxuekai@ahnu.edu.cn (X.J.); wfcxi17@mail.ahnu.edu.cn (W.C.); sunjy228@mail.ahnu.edu.cn (J.S.) \\ * Correspondence: liuyizhang111@126.com (Y.L.); fgao@mail.ahnu.edu.cn (F.G.); Tel.: +86-553-3937137 (F.G.)
}

Received: 15 November 2018; Accepted: 27 November 2018; Published: 5 December 2018

check for updates

The authors wish to make the following corrections to their paper [1]:

The author Yizhang Liu's affiliations were incorrect in our published paper in Sensors [1]. Therefore, they are corrected from

"( ${ }^{1}$ Department of Food and Environmental Engineering, Vocational and Technical College, Chuzhou 239001, China

${ }^{2}$ Laboratory of Functionalized Molecular Solids, Ministry of Education, Anhui Key Laboratory of Chemo/Biosensing, Laboratory of Optical Probes and Bioelectrocatalysis (LOPAB), College of Chemistry and Materials Science, Anhui Normal University, Wuhu 241000, China)" to

" ${ }^{1}$ Department of Food and Environmental Engineering, Chuzhou Vocational and Technical College, Chuzhou 239001, China

${ }^{2}$ Laboratory of Functionalized Molecular Solids, Ministry of Education, Anhui Key Laboratory of Chemo/Biosensing, Laboratory of Optical Probes and Bioelectrocatalysis (LOPAB), College of Chemistry and Materials Science, Anhui Normal University, Wuhu 241000, China)".

The manuscript will be updated and the original will remain online on the article webpage.

The authors would like to apologize for any inconvenience caused.

Conflicts of Interest: The authors declare no conflicts of interest.

\section{Reference}

1. Liu, Y.; Jiang, X.; Cao, W.; Sun, J.; Gao, F. Detection of Thrombin Based on Fluorescence Energy Transfer between Semiconducting Polymer Dots and BHQ-Labelled Aptamers. Sensors 2018, 18, 589. [CrossRef] [PubMed]

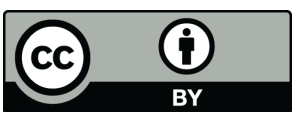

(C) 2018 by the authors. Licensee MDPI, Basel, Switzerland. This article is an open access article distributed under the terms and conditions of the Creative Commons Attribution (CC BY) license (http:/ / creativecommons.org/licenses/by/4.0/). 